\title{
Prior housing conditions and sleep loss may affect recovery from brain injury in rats: A pilot study
}

\author{
Ronald G. Riechers, MD; ${ }^{1-2}$ Jaime L. Shuster, MA; ${ }^{1,3}$ Kathryn J. Bryan, PhD; ${ }^{1}$ Christopher J. Burant, PhD; ${ }^{4-5}$ \\ Sherry L. Ball, PhD ${ }^{1,3,6 *}$ \\ ${ }^{1}$ Louis Stokes Cleveland Department of Veterans Affairs Medical Center (VAMC), Cleveland, OH; ${ }^{2}$ Department of \\ Neurology, Case Western Reserve University, Cleveland, $\mathrm{OH} ;{ }^{3}$ Department of Psychology, Cleveland State University, \\ Cleveland, $\mathrm{OH} ;{ }^{4}$ Geriatric Research, Education and Clinical Center, Louis Stokes Cleveland VAMC, Cleveland, $\mathrm{OH}$; \\ ${ }^{5}$ Frances Payne Bolton School of Nursing, Case Western Reserve University, Cleveland, $\mathrm{OH} ;{ }^{6}$ Department of \\ Ophthalmology, The Cleveland Clinic Lerner College of Medicine of Case Western Reserve University, Cleveland, $\mathrm{OH}$
}

\begin{abstract}
The purpose of this study is to understand the effect of combat-associated conditions such as sleep deprivation (SD) on subsequent traumatic brain injury (TBI). Prior to TBI (or sham surgery) induced by controlled cortical impact $(\mathrm{CCI})$, rats were housed singly in chambers that prevented rapid eye movement sleep or allowed unrestricted sleep (no $\mathrm{SD}$ ). Sensorimotor function was tested pre-SD and retested on postoperative days (PDs) 4, 7, and 14. Two additional control groups were housed socially prior to either CCI or sham surgery. CCI resulted in immediate performance deficits on sensorimotor tasks. The $\mathrm{PD}$ on which performance returned to baseline depended on preinjury conditions. Overall, preinjury $\mathrm{SD}+\mathrm{CCI}$ resulted in an earlier recovery than no $\mathrm{SD}+\mathrm{CCI}$, and the no $\mathrm{SD}+\mathrm{CCI}$ group (housed singly under conditions comparable with the SD group) recovered slower than all other groups. These data are the first to raise the possibility that recovery of sensorimotor function following TBI is affected by preinjury conditions. The data suggest that preinjury SD $24 \mathrm{~h}$ in duration may result in faster recovery and that novel or social isolation conditions may impede recovery. Thus, the combat environment may contribute to complexities associated with TBIs common in U.S. servicemembers.
\end{abstract}

Key words: combat, controlled cortical impact, novel housing, premorbid conditions, sensorimotor function, sleep deprivation, social isolation, stress, trauma, traumatic brain injury.

\section{INTRODUCTION}

Traumatic brain injury (TBI) has been labeled the signature injury of the conflicts in Iraq and Afghanistan [1-2]. While TBIs experienced by servicemembers in the combat theater are variable, several aspects of the environment are constant. Combat operations are associated with high stress and prolonged periods of sleep deprivation (SD) [3-4]. However, most clinical studies of TBI have been in populations where the environment is relatively constant and secure [5]. Individuals with sportsrelated TBI are often participating in activities for which they are typically well rested and they have physiologically and psychologically prepared their bodies [6-7].

\footnotetext{
Abbreviations: $\mathrm{ANOVA}=$ analysis of variance, $\mathrm{CCI}=$ controlled cortical impact, $\mathrm{NH}=$ normal housing, $\mathrm{PBS}=$ phosphatebuffered saline, $\mathrm{PD}=$ postoperative day, REM = rapid eye movement, $\mathrm{SD}=$ sleep deprivation, $\mathrm{TBI}=$ traumatic brain injury.

*Address all correspondence to Sherry L. Ball, PhD; Research Service, 151W, Louis Stokes Cleveland VAMC, Cleveland, OH 44106; 216-791-3800, ext 5278; fax: 216229-8509. Email: sherry.ball@case.edu http://dx.doi.org/10.1682/JRRD.2012.04.0061
} 
While members of this population are relatively homogeneous in nature and similar in age to the typical servicemember, they diverge from the servicemember in the injury environment, background physical state, and stress level [6].

Stress [8-9] and SD [10-11] have known effects on nervous system function of both humans [12] and animals [13]. Our pilot study sought to better define the effects of preinjury SD on recovery in an animal model of TBI. Animal models of SD have demonstrated altered glucose metabolism [14], increased pro-inflammatory cytokine production [15], abnormal neuronal excitability [16-17], and altered thermoregulation [18], all of which theoretically could be associated with increased pathology and behavioral deficits following TBI. Changes in glucose metabolism could increase tissue $\mathrm{pH}$ and place greater oxidative stress on the injured brain [19]. Altered thermoregulation with increases in temperature could also be deleterious to the injured brain [20]. Additionally, in animal models, SD yields significant changes in gene expression and neurogenesis [21-22], which could dramatically affect the injury response. Finally, both genetics [23-24] and biochemical changes induced by stressful conditions [25] are thought to have an effect on TBI severity. Combining validated models for rapid eye movement (REM) SD and TBI allows for a better understanding of the effect of the combat lifestyle on the quintessential combat injury. While other studies [26] have considered the effect of stress following injury, this is the first study to assess the effect of preinjury conditions. Although pre-injury conditions are virtually inaccessible in terms of treatment, the nature of their effect on disease progression may prove elemental to optimizing treatment strategies.

\section{METHODS}

We used 32 male Long Evans Blue Spruce rats (72$78 \mathrm{~d}$ of age and $250-315 \mathrm{~g}$, Harlan Laboratories; Indianapolis, Indiana). Before experimental manipulation, we housed all animals socially with food and water available ad libitum.

We trained the rats daily for $5 \mathrm{~d}$ on both beam walk and adhesive removal tasks $7 \mathrm{~d}$ prior to performing controlled cortical impact (CCI) surgery to induce TBI. For the beam walk task, we trained the rats to traverse a $1.0 \mathrm{~m}$ long narrow beam (width: $2.0 \mathrm{~cm}$ ) elevated $90 \mathrm{~cm}$ for five trials per day. On day 5 of training, we recorded baseline performance measures. All rats were preoperatively able to transverse the beam without footslips and enter a goal box at the end of the beam. We allowed rats to stay in the goal box for $30 \mathrm{~s}$ before returning them to their home cage. We retested the rats on the beam on postoperative days (PDs) 4,7 , and 14 . We recorded the time spent traversing the beam and distances reached and calculated speed as distance/time. We motivated the rats to cross the beam to escape white noise aversive stimuli and to receive a treat (8in1 Yogies for Rats, Ecotrition, United Pet Group; Cincinnati, Ohio). However, since after surgery all the rats fell off the beam before reaching the goal box, we made performance comparisons by assessing speed rather than time to reach the goal box. We placed the rats in their home cage for 2 min between each trial.

For the adhesive tab removal task, training also began $7 \mathrm{~d}$ prior to surgery and lasted $5 \mathrm{~d}$. We trained rats to remove a round $(0.75$ in.) adhesive sticker (no. 5466, Avery; Brea, California) from the distal radial surface of each forelimb. On day 5 of training, we recorded baseline performance. We administered two trials per forelimb $5 \mathrm{~min}$ apart and terminated each trial when the adhesive tab was removed or 2 min had elapsed. We recorded latencies to remove each tab $3 \mathrm{~d}$ prior to injury and on PDs 4, 7, and 14 .

On preoperative day 3 , we completed training and baseline sensorimotor function assessment and randomly assigned the rats to one of four experimental groups: (1) $24 \mathrm{~h}$ of SD followed by CCI (SD+CCI group), (2) $24 \mathrm{~h}$ of housing in platform over water chambers with no SD followed by CCI (no SD+CCI group), (3) normal housing ( $\mathrm{NH}$; social housing in standard rodent cages) before $\mathrm{CCI}(\mathrm{NH}+\mathrm{CCI}$ group), or (4) $\mathrm{NH}$ before sham surgery ( $\mathrm{NH}+$ sham group).

One day prior to surgery, we moved the rats in the $\mathrm{SD}+\mathrm{CCI}$ and no $\mathrm{SD}+\mathrm{CCI}$ groups from $\mathrm{NH}$ conditions to single housing in chambers with small or large platforms, respectively. We housed the $\mathrm{SD}+\mathrm{CCI}$ group rats singly on a small platform (diameter: $10.0 \mathrm{~cm}$ ) secured to a base placed in an opaque plastic 5 gal container at an elevation of $10.16 \mathrm{~cm}$ from the bottom of the container. Water filled the container to a level surrounding the platform. The small platform was large enough to support the rat when it was awake but too small to support the body and head while it was asleep. When the rat entered REM sleep and lost muscle tone, the rat's head would fall into the water and wake it. Preinjury housing for the no SD+CCI group was the same as the SD+CCI group, except the platform was large enough (diameter: $14.0 \mathrm{~cm}$ ) to allow the rat to 
enter REM sleep without contacting the water [27]. Food and water were available ad libitum to the rats while they were in the platform over water conditions.

At the end of the $24 \mathrm{~h} \mathrm{SD}$ and no SD period, the rats underwent a TBI via CCI while the NH rats received either a CCI or a sham surgery. We used the CCI model of TBI to produce a unilateral injury to the sensorimotor cortex. We collected sensorimotor performance measures on PDs 4, 7, and 14. On PD 15, we intracardially perfused the rats with phosphate-buffered saline (PBS) containing 4 percent paraformaldehyde. We removed the brains and postfixed them in 4 percent paraformaldehyde for $24 \mathrm{~h}$, followed by incubation in cryoprotectant. We collected one $50 \mu \mathrm{m}$ frozen section every $200 \mu \mathrm{m}$ between $+1.1 \mathrm{~mm}$ and $-3.8 \mathrm{~mm}$ relative to bregma.

We conducted all surgical procedures using aseptic conditions. We placed the rats on a $37^{\circ} \mathrm{C}$ heating pad to maintain a physiologically normal body temperature and anesthetized them with 4 percent isoflurane in a mixture of 30 percent nitrous oxide and 70 percent oxygen delivered through a nose cone and mounted in a stereotaxic device, with their heads fixed in a horizontal position throughout the procedure. We used a high-speed dental drill to create a $5.0 \mathrm{~mm}$ craniotomy positioned at bregma and $2.0 \mathrm{~mm}$ from midline over the left sensorimotor cortex, keeping the dura intact. We used a probe (diameter: $3.0 \mathrm{~mm}$ ) attached to an impact device (My Neurolab; St. Louis, Missouri) to compress the brain a depth of $2.0 \mathrm{~mm}$ at a speed of $3.0 \mathrm{~m} / \mathrm{s}$ in order to cause mild to moderate trauma [28]. Sham-injured rats received the same anesthesia and craniotomy but were not subjected to TBI. After we sutured the scalp, we allowed all rats to recover from surgery on a heating pad maintained at $37^{\circ} \mathrm{C}$. In all cases, the surgeon was blinded to the treatment status of the rat. We administered buprenorphine, an analgesic, at a dosage of $0.05 \mathrm{mg} / \mathrm{kg}$ subcutaneously for $24 \mathrm{~h}$ postoperatively.

On PD 15, we gave the rats a lethal overdose of sodium pentobarbital followed by cardiac perfusion. We collected the brains and postfixed them for $24 \mathrm{~h}$ in 4 percent paraformaldehyde followed by cryoprotection in 30 percent sucrose in PBS overnight. We prepared coronal brain sections $50 \mu \mathrm{m}$ in thickness and stained them with cresyl violet. We took digital images of one section every $500 \mu \mathrm{m}$ and analyzed percent lesion size by tracing the entire area of each hemisphere and calculating the percent area of the ipsilateral as compared with the contralateral hemisphere area using ImageJ (National Institutes of Health; Bethesda, Maryland).
We conducted a repeated-measures analysis of variance (ANOVA) (group $\times$ day) on the data to assess beam walk speeds and latency to remove adhesive tabs. Raw adhesive tab removal latencies were log-transformed because of lack of sphericity (Mauchly $p<0.01$ ). We conducted separate pairwise comparisons between baseline and PDs 4, 7, and 14 for each group, controlling for family-wise error rate across the tests at 0.05 level based on Bonferonni post hoc tests. For lesion volume, we conducted a one-way ANOVA. We calculated all tests using standard procedures (SPSS version 17.0 for Windows, IBM Corporation; Armonk, New York). We used a significance level of $p<0.05$ for all statistical analyses.

\section{RESULTS}

We examined the effects of preinjury SD and no SD on recovery of hind-limb motor function using a modified beam walk task; results are plotted in Figure 1. For each test

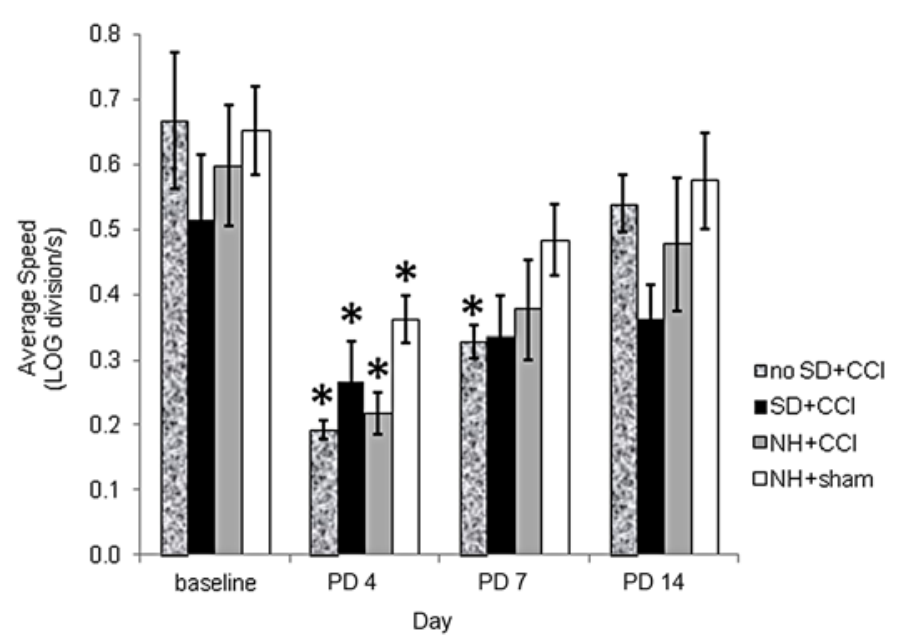

Figure 1.

Performance on beam walk motor task before and after traumatic brain injury or sham surgery. On postoperative day (PD) 3 , performance deficits were noted in all groups; all groups had significant decreases when compared with baseline measures. By PD 7, performance was comparable with baseline levels in controlled cortical impact (CCl) groups (sleep deprivation $[\mathrm{SD}]+\mathrm{CCl}$ and normal housing $[\mathrm{NH}]+\mathrm{CCl}$ ) but not no SD+CCl group. For no $\mathrm{SD}+\mathrm{CCl}$ group, beam crossing speed was significantly slower on PD 7 than baseline. By PD 14, all groups performed at levels comparable with baseline measures. Error bars represent standard error. * Significantly different from baseline $(p<0.05)$. 
day (baseline and PDs 4, 7, and 14), we calculated beam speed as the distance traveled on the beam divided by time to reach the goal box or fall from the beam. There was a significant effect of day (Greenhouse-Geisser $F(1.64,37.81)=$ $29.99, p<0.001)$. All rats were able to traverse the length of the beam at comparable speeds (no significant differences) at baseline. Following CCI, however, few rats were able to reach the goal box before falling off the beam, making the distance each rat traveled variable. Thus, we presented these data as speed measurements rather than latency. We conducted separate pairwise comparisons using $t$-tests between baseline and PDs 4, 7, and 14 for each group. Based on $t-$ tests, beam speeds were significantly slower between baseline and PD 4 for all groups (SD+CCI: $t(6)=5.67, p=0.01$; no SD+CCI: $t(5)=4.89, p<0.01$; NH+CCI: $t(5)=5.51, p<$ 0.01 ; $\mathrm{NH}+$ sham: $t(7)=4.18, p<0.01)$. We expected no effect of surgery on performance in the $\mathrm{NH}+$ sham group. At PD 4, however, there appeared to be a significant effect of the craniotomy alone as has been reported by others [29]. On PD 7, the no SD+CCI group remained significantly $(t(5)=3.18, p<0.05)$ slower than baseline speeds, while we found no significant differences in speed comparing baseline and PD 7 for any other group. This suggests that recovery had occurred by PD 7 in all groups except the no $\mathrm{SD}+\mathrm{CCI}$ group. Also, we found no significant differences in speed for any groups between baseline and PD $14(p>$ $0.05)$. There was no interaction between group $\times$ day (Greenhouse-Geisser $F(4.93,37.8)=0.996, p>0.05)$. There were no significant differences between groups $(F(1.88)=3$, $p>0.05)$. Speeds gradually increased from PD 4 to 14 for all groups, with all groups reaching speeds comparable with baseline by PD 14 ( $p>0.05)$.

We assessed forelimb sensorimotor function using the adhesive tab removal task; data are plotted in Figure 2. These data revealed a significant effect of day (Greenhouse-Geisser $F(2.04,36.7)=5.28, p<0.01$ ) and between groups $(F(3,18)=3.38, p<0.05)$, but no significant interaction for group $\times$ day $(F(6.1,24)=1.73, p>$ $0.05)$. Again, we conducted separate pairwise comparisons using $t$-tests of average tab removal latencies between baseline and PDs 4, 7, and 14 for each group, controlling for family-wise error rate across the tests at the 0.05 level based on Bonferroni post hoc tests. We expected that practice performance on the tab removal task might improve or that latency to remove would decrease. Rats with CCI showed no improvements in performance on any PD. However, rats with sham surgery only did show improved performance postoperatively.

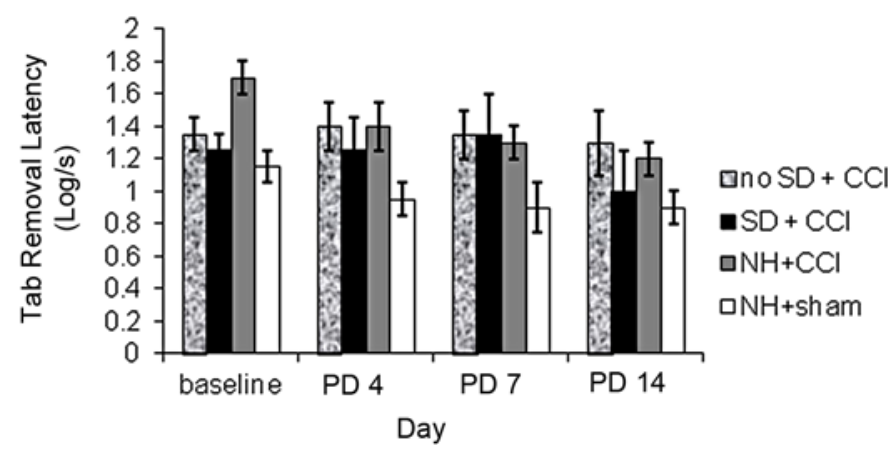

Figure 2.

Performance on adhesive tab removal before and after traumatic brain injury ( $\mathrm{TBI}$ ) or sham surgery. Time to remove tabs was not significantly affected by TBI or sham surgery only. Statistical analysis revealed that on each postoperative day (PD), time latencies to remove both tabs were comparable with baseline levels for each experimental group. Error bars represent standard error. $\mathrm{CCl}=$ controlled cortical impact, $\mathrm{NH}=$ normal housing, SD = sleep deprivation.

On PD 4, the $\mathrm{NH}+$ sham group performed significantly $(t(6)=12.94, p<0.01)$ better than baseline, suggesting a CCI injury-induced deficit on performance; there were no significant differences between baseline and PD 4 for the $\mathrm{SD}+\mathrm{CCI}$, no $\mathrm{SD}+\mathrm{CCI}$, or $\mathrm{NH}+\mathrm{CCI}$ groups. However, the $\mathrm{NH}+\mathrm{CCI}$ group removed tabs significantly faster on $\mathrm{PD}$ 14 than at baseline $(t(6)=2.49, p<0.05)$. We found no significant differences between baseline and PD 7 for any group and no significant differences between baseline and PD 14 for the $\mathrm{SD}+\mathrm{CCI}$, no $\mathrm{SD}+\mathrm{CCI}$, or $\mathrm{NH}+$ sham groups $(p>0.05)$. Thus, no improvements from baseline levels occurred on any PD for the SD+CCI and the no SD+CCI groups, while performance improvement occurred on $\mathrm{PD}$ 4 for the $\mathrm{NH}+$ sham group and on $\mathrm{PD} 14$ for the $\mathrm{NH}+\mathrm{CCI}$ group. While results from sham surgery rats revealed temporary effects, at least one other study reported that effects remained at PD 14 [29].

These trends are similar to those noted with hindlimb function. Although latency averages are similar between all groups by PD 14, recovery patterns were different for individual groups. The SD+CCI group had a slight but not significant increase in latency or poorer performance on PDs 4 and 7 than at baseline, while the overall trend for all other groups was a decrease in latency or performance improvement on each PD. Sham surgery rats improved by PD 4, while the $\mathrm{NH}+\mathrm{CCI}$ group improved at PD 14. This suggests that practice effects 
had already resulted in performance improvement in the sham surgery rats on PD 4, while performance improvements in the $\mathrm{NH}+\mathrm{CCI}$ group did not occur until PD 14; we observed no significant performance improvement in either the SD or no SD rats.

Calculated percent lesion volumes (ipsilateral hemispheric volume divided by the contralateral hemispheric volume $\times 100$ ) revealed comparable injury sizes between all CCI groups and no measurable lesions present in shamonly rats (Figure 3). Based on Bonferroni post hoc tests $(F(3,20)=8.251, p<0.001)$, the $\mathrm{NH}+$ sham group was significantly different from all other groups. We found no significant differences among the three other CCI groups.

\section{DISCUSSION}

These data support the hypothesis that preinjury conditions can affect recovery from TBI. We saw a somewhat unexpected protective effect of SD on the acute injuryinduced effects for hind-limb motor function in this model. Despite the known and well-described neurophysiologic effects of SD, of all the groups receiving a CCI, the SD

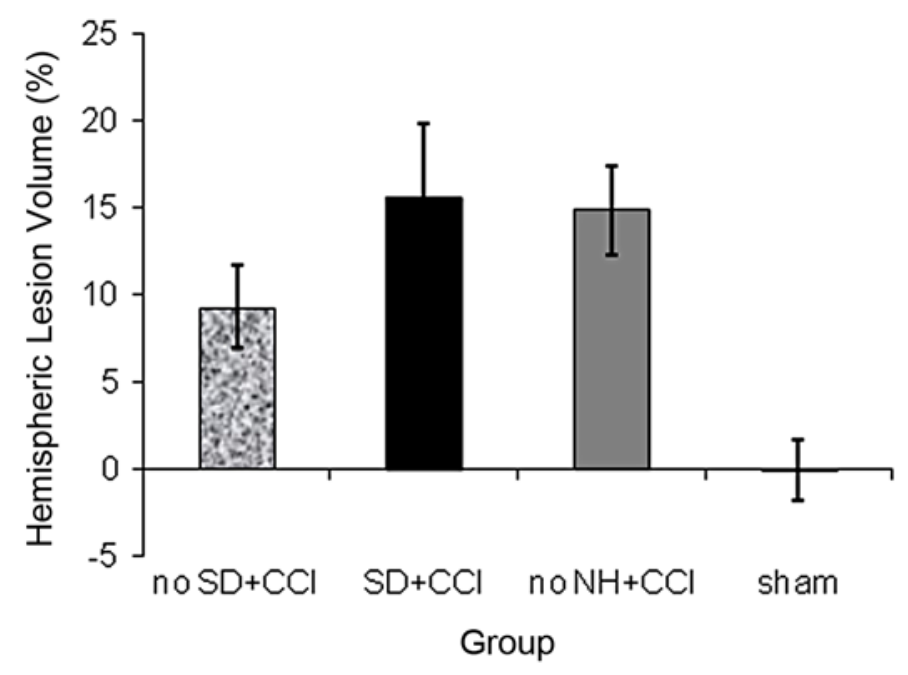

Figure 3.

Percent lesion volume for all experimental groups. Average lesion size for each group calculated from cresyl violet-stained sections through entire lesioned area. Sham-only group was significantly different from all groups $(p<0.01)$. No significant differences were found among three other controlled cortical impact $(\mathrm{CCl})$ groups. Error bars represent standard error. $\mathrm{NH}=$ normal housing, SD = sleep deprivation. group had the least decline in beam walking speeds and the no SD+CCI group had the greatest decline in beam walking speeds. Both the no $\mathrm{SD}+\mathrm{CCI}$ and $\mathrm{SD}+\mathrm{CCI}$ groups experienced the stress of novel housing conditions, including social isolation. However, the SD group additionally experienced SD but still performed better than the no $\mathrm{SD}+\mathrm{CCI}$ group on the beam walk task. This further supports the possibility of a neuroprotective effect. On the sensorimotor task of adhesive tab removal, the data did not show the same neuroprotective effects but also did not show statistical evidence of worsening. In fact, while the $\mathrm{NH}+$ sham and $\mathrm{NH}+\mathrm{CCI}$ groups both showed performance improvements over baseline on PDs 14 and 4, respectively, the $\mathrm{SD}+\mathrm{CCI}$ and no SD+CCI groups showed no improvements over baseline on any PD. To further investigate the unexpected neuroprotective effect, future experiments will include longer SD durations.

Analyzing sham surgery (craniotomy only) rats revealed an unexpected effect on behavioral measures, most likely because of the induction of a neurovascular injury as has been proposed by others [29]. Mechanical stress placed on the surface of the brain during drilling to create the bone flap could have triggered injury response mechanisms that led to secondary injury responses, resulting in a localized loss of function. Future studies will include additional control groups without craniotomy. A thorough analysis of brain integrity following craniotomy should be considered, as well as the inclusion of anesthesia-only rats as controls, for future studies.

The mechanisms for this potential neuroprotective effect are not clearly established, but future studies should seek to further validate trends demonstrated in our pilot study and better understand the neurochemical changes herein. It will be especially intriguing to investigate the underlying mechanisms of the potential neuroprotective properties of $24 \mathrm{~h}$ of SD. The trend for the no SD+CCI group to exhibit performance deficits may be related to the potentially stressful novel environment and social isolation conditions associated with the individual platform over water chambers. Repeated physical exercise has also been shown to diminish the harmful effects of social isolation, as demonstrated by changes to intracellular glucocorticoid receptor and its nuclear transporter protein 70 [30]. There is no question that under social isolation, novel environment, $\mathrm{SD}$, or other potentially stressful conditions, the neurochemical milieu of the brain becomes altered. Adenosine, a ubiquitous neuromodulator, plays a key role in these changes by accumulating both during wakefulness and in 
response to ischemia, hypoxia, excitotoxicity, inflammation, and other types of TBI [31]. Interestingly, an agent that has significant effect on adenosine neurochemistry, caffeine, has also been demonstrated to exert neuroprotective effects after stroke [32] and other neurologic conditions [33-34]. The fact that one study found neuroprotective effects of caffeine when given chronically but not acutely [34] suggests that acute elevation of adenosine as seen with $24 \mathrm{~h}$ of SD may but protective, while longer duration SD may be detrimental to brain function. This constellation of information raises the possibility that adenosine may also play a role in the potential neuroprotective effect of REM SD.

In humans, mild TBI typically produces no permanent morphological or neurological deficits, while cognitive deficits may persist for years following the initial injury [35]. Severe injury in humans is conventionally considered to be associated with a period of unconsciousness or coma that lasts for $\geq 2 \mathrm{~d}$ and chronic neurological defects [36]. Although loss of consciousness cannot be compared between rat and human studies because injuries are performed under anesthesia, neurological and cognitive deficits provide a means for comparison. This protocol uses the CCI injury model originally developed by Dixon et al. [28]. Using this model, Dixon et al. defined injuries resulting from compression depths of 1,2 , or $3 \mathrm{~mm}$ as low, moderate, or severe and as comparable with mild, moderate, and severe TBI as defined in human patients [28,37].

Future studies should evaluate the effect of longer periods of REM SD and determine whether longer duration REM SD will lead to greater neuroprotective effects, or conversely, increased central nervous system damage. Use of multiplatform REM SD chambers will help to dissect at least the effects of social isolation. More sensitive measures of sensorimotor performance, particularly the use of behavioral tasks that are less sensitive to practice effects, may prove useful and may yield clearer results. Any effect of preinjury conditions on cognitive function would be particularly relevant to understanding the etiology of TBI in returning servicemembers. Additionally, the role of total SD has also yet to be evaluated and its effect on TBI should also be studied.

\section{CONCLUSIONS}

Although the effect reported in this study is mild, the implications may be quite significant. Understanding the unique effects involved in TBI incurred under common combat conditions is needed in order to generate a better animal model that considers the environment of the servicemember at the time of TBI. As new treatment strategies evolve, it will be imperative to consider their effectiveness in a model that includes these factors. Despite the plethora of data on alterations in cell death, inflammation, oxidative stress, and neurotransmitter systems following TBI, no treatments have proven to be clinically effective in improving mortality or limiting disability following injury [38]. It is possible that the condition of the combat servicemember is fundamentally different from the animal model and that the development of optimally effective treatments for combat-associated TBI depends on examining antecedent conditions.

Future studies should address the effect of chronic levels of SD, which more closely align with the combat servicemember's experience. Additionally, emotional and cognitive function should be evaluated in addition to sensorimotor function. While this and numerous other studies have shown temporary effects of TBI on sensorimotor function, it is possible that deficits in emotional and cognitive function could be more long-lasting.

\section{ACKNOWLEDGMENTS}

\section{Author Contributions:}

Study concept and design: R. G. Riechers, S. L. Ball, J. L. Shuster. Acquisition of data: J. L. Shuster.

Analysis and interpretation of data: K. J. Bryan, S. L. Ball,

R. G. Riechers.

Drafting of manuscript: R. G. Riechers, S. L. Ball.

Critical revision of manuscript for important intellectual content:

K. J. Bryan, R. G. Riechers, S. L. Ball.

Statistical analysis: C. J. Burant, S. L. Ball.

Obtained funding: R. G. Riechers, S. L. Ball.

Financial Disclosures: The authors have declared that no competing interests exist.

Funding/Support: This material was based on work supported by the Department of Veterans Affairs Office of Rehabilitation Research and Development and the Cleveland Veterans Health Administration Medical Research and Education Foundation (to Drs. Riechers and Ball).

Additional Contributions: The authors are grateful for support and assistance provided by the Louis Stokes Cleveland Department of Veterans Affairs Medical Center research service office and to Brett Hanzlicek for technical assistance. Jaime L. Shuster, $\mathrm{MA}, \mathrm{PhD}$, is now Assistant Professor of Psychology at Eastern Gateway Community College, Steubenville, Ohio.

Institutional Review: All animal procedures were approved by the local Institutional Animal Care and Use Committee of the Louis Stokes Cleveland Department of Veterans Affairs Medical Center and performed in accordance with standards prescribed by the Society for Neuroscience. 


\section{REFERENCES}

1. Hoge CW, McGurk D, Thomas JL, Cox AL, Engel CC, Castro CA. Mild traumatic brain injury in U.S. Soldiers returning from Iraq. N Engl J Med. 2008;358(5):453-63. [PMID:18234750] http://dx.doi.org/10.1056/NEJMoa072972

2. Warden DL, Salazar AM, Martin EM, Schwab KA, Coyle M, Walter J; The DVHIP Study Group. A home program of rehabilitation for moderately severe traumatic brain injury patients. J Head Trauma Rehabil. 2000;15(5):1092-1102. [PMID:10970930] http://dx.doi.org/10.1097/00001199-200010000-00003

3. Miller NL, Shattuck LG, Matsangas P. Sleep and fatigue issues in continuous operations: a survey of U.S. Army officers. Behav Sleep Med. 2011;9(1):53-65. [PMID:21218294] http://dx.doi.org/10.1080/15402002.2011.533994

4. Lieberman HR, Bathalon GP, Falco CM, Kramer FM, Morgan CA 3rd, Niro P. Severe decrements in cognition function and mood induced by sleep loss, heat, dehydration, and undernutrition during simulated combat. Biol Psychiatry. 2005;57(4):422-29. [PMID:15705359] http://dx.doi.org/10.1016/j.biopsych.2004.11.014

5. Loane DJ, Faden AI. Neuroprotection for traumatic brain injury: translational challenges and emerging therapeutic strategies. Trends Pharmacol Sci. 2010;31(12):596-604. [PMID:21035878] http://dx.doi.org/10.1016/j.tips.2010.09.005

6. Meyer KS, Marion DW, Coronel H, Jaffee MS. Combatrelated traumatic brain injury and its implications to military healthcare. Psychiatr Clin North Am. 2010;33(4):783-96. [PMID:21093678] http://dx.doi.org/10.1016/j.psc.2010.08.007

7. Ray SK, Dixon CE, Banik NL. Molecular mechanisms in the pathogenesis of traumatic brain injury. Histol Histopathol. 2002;17(4):1137-52. [PMID:12371142]

8. Pêgo JM, Sousa JC, Almeida OF, Sousa N. Stress and the neuroendocrinology of anxiety disorders. Curr Top Behav Neurosci. 2010;2:97-117. [PMID:21309108] http://dx.doi.org/10.1007/7854_2009 13

9. McEwen BS. Protection and damage from acute and chronic stress: allostasis and allostatic overload and relevance to the pathophysiology of psychiatric disorders. Ann N Y Acad Sci. 2004;1032:1-7. [PMID:15677391] http://dx.doi.org/10.1196/annals.1314.001

10. Cirelli C, Tononi G. Molecular neurobiology of sleep. Handb Clin Neurol. 2011;98:191-203. [PMID:21056187] http://dx.doi.org/10.1016/B978-0-444-52006-7.00012-5

11. McEwen BS. Sleep deprivation as a neurobiologic and physiologic stressor: Allostasis and allostatic load. Metabolism. 2006;55(10 Suppl 2):S20-23. [PMID:16979422] http://dx.doi.org/10.1016/j.metabol.2006.07.008
12. Nishino S. Hypothalamus, hypocretins/orexin, and vigilance control. Handb Clin Neurol. 2011;99:765-82.

[PMID:21056227]

http://dx.doi.org/10.1016/B978-0-444-52007-4.00006-0

13. Everson CA. Functional consequences of sustained sleep deprivation in the rat. Behav Brain Res. 1995;69(1-2):43-54. [PMID:7546317] http://dx.doi.org/10.1016/0166-4328(95)00009-I

14. Knutson KL, Spiegel K, Penev P, Van Cauter E. The metabolic consequences of sleep deprivation. Sleep Med Rev. 2007;11(3):163-78. [PMID:17442599] http://dx.doi.org/10.1016/j.smrv.2007.01.002

15. van Leeuwen WM, Lehto $M$, Karisola P, Lindholm H, Luukkonen R, Sallinen M, Härmä M, Porkka-Heiskanen T, Alenius H. Sleep restriction increases the risk of developing cardiovascular diseases by augmenting proinflammatory responses through IL-17 and CRP. PLoS ONE. 2009; 4(2):e4589. [PMID:19240794] http://dx.doi.org/10.1371/journal.pone.0004589

16. Kreuzer P, Langguth B, Popp R, Raster R, Busch V, Frank E, Hajak G, Landgrebe M. Reduced intra-cortical inhibition after sleep deprivation: a transcranial magnetic stimulation study. Neurosci Lett. 2011;493(3):63-66. [PMID:21352891] http://dx.doi.org/10.1016/j.neulet.2011.02.044

17. Yan J, Li JC, Xie ML, Zhang D, Qi AP, Hu B, Huang W, Xia JX, Hu ZA. Short-term sleep deprivation increases intrinsic excitability of prefrontal cortical neurons. Brain Res. 2011;1401:52-58. [PMID:21663896]

http://dx.doi.org/10.1016/j.brainres.2011.05.032

18. Parmeggiani PL. Interaction between sleep and thermoregulation: an aspect of the control of behavioral states. Sleep. 1987;10(5):426-35. [PMID:3317725]

19. Auer RN. Hypoglycemic brain damage. Metab Brain Dis. 2004;19(3-4):169-75. [PMID:15554413] http://dx.doi.org/10.1023/B:MEBR.0000043967.78763.5b

20. Wang CX, Stroink A, Casto JM, Kattner K. Hyperthermia exacerbates ischaemic brain injury. Int J Stroke. 2009;4(4): 274-84. [PMID:19689756]

http://dx.doi.org/10.1111/j.1747-4949.2009.00317.x

21. Robinson SA, Loiacono RE, Christopoulos A, Sexton PM, Malone DT. The effect of social isolation on rat brain expression of genes associated with endocannabinoid signaling. Brain Res. 2010;1343:153-67. [PMID:20430015] http://dx.doi.org/10.1016/j.brainres.2010.04.031

22. Stranahan AM, Khalil D, Gould E. Social isolation delays the positive effects of running on adult neurogenesis. Nat Neurosci. 2006;9(4):526-33. [PMID:16531997] http://dx.doi.org/10.1038/nn1668

23. Dalla Libera AL, Regner A, de Paoli J, Centenaro L, Martins TT, Simon D. IL-6 polymorphism associated with fatal outcome in patients with severe traumatic brain injury. Brain Inj. 2011;25(4):365-69. [PMID:21314275] http://dx.doi.org/10.3109/02699052.2011.556107 
24. Waters RJ, Nicoll JA. Genetic influences on outcome following acute neurological insults. Curr Opin Crit Care. 2005;11(2):105-10. [PMID:15758588] http://dx.doi.org/10.1097/01.ccx.0000155354.78617.91

25. Cooper DB, Kennedy JE, Cullen MA, Critchfield E, Amador RR, Bowles AO. Association between combat stress and post-concussive symptom reporting in OEF/OIF service members with mild traumatic brain injuries. Brain Inj. 2011;25(1):1-7. [PMID:21117916] http://dx.doi.org/10.3109/02699052.2010.531692

26. Griesbach GS, Hovda DA, Tio DL, Taylor AN. Heightening of the stress response during the first weeks after a mild traumatic brain injury. Neuroscience. 2011;178:147-58. [PMID:21277947] http://dx.doi.org/10.1016/j.neuroscience.2011.01.028

27. Mendelson WB, Guthrie RD, Frederick G, Wyatt RJ. The flower pot technique of rapid eye movement (REM) sleep deprivation. Pharmacol Biochem Behav. 1974;2(4):553-56. [PMID:4371007] http://dx.doi.org/10.1016/0091-3057(74)90018-5

28. Dixon CE, Clifton GL, Lighthall JW, Yaghmai AA, Hayes RL. A controlled cortical impact model of traumatic brain injury in the rat. J Neurosci Methods. 1991;39(3):253-62. [PMID:1787745] http://dx.doi.org/10.1016/0165-0270(91)90104-8

29. Cole JT, Yarnell A, Kean WS, Gold E, Lewis B, Ren M, McMullen DC, Jacobowitz DM, Pollard HB, O'Neill JT, Grunberg NE, Dalgard CL, Frank JA, Watson WD. Craniotomy: true sham for traumatic brain injury, or a sham of a sham? J Neurotrauma. 2011;28(3):359-69. [PMID:21190398] http://dx.doi.org/10.1089/neu.2010.1427

30. Filipović D, Gavrilović L, Dronjak S, Radojcić MB. The effect of repeated physical exercise on hippocampus and brain cortex in stressed rats. Ann N Y Acad Sci. 2007; 1096:207-19. [PMID:17405932] http://dx.doi.org/10.1196/annals.1397.087

31. Pedata F, Corsi C, Melani A, Bordoni F, Latini S. Adenosine extracellular brain concentrations and role of A2A receptors in ischemia. Ann N Y Acad Sci. 2001;939:74-84. [PMID:11462806] http://dx.doi.org/10.1111/j.1749-6632.2001.tb03614.x

32. Zhao X, Liu SJ, Zhang J, Strong R, Aronowski J, Grotta JC. Combining insulin-like growth factor derivatives plus caffeinol produces robust neuroprotection after stroke in rats. Stroke. 2005;36(1):129-34. [PMID:15569874] http://dx.doi.org/10.1161/01.STR.0000149624.87661.18

33. Kalda A, Yu L, Oztas E, Chen JF. Novel neuroprotection by caffeine and adenosine $\mathrm{A}(2 \mathrm{~A})$ receptor antagonists in animal models of Parkinson's disease. J Neurol Sci. 2006; 248(1-2):9-15. [PMID:16806272] http://dx.doi.org/10.1016/j.jns.2006.05.003
34. Li W, Dai S, An J, Li P, Chen X, Xiong R, Liu P, Wang H, Zhao Y, Zhu M, Liu X, Zhu P, Chen JF, Zhou Y. Chronic but not acute treatment with caffeine attenuates traumatic brain injury in the mouse cortical impact model. Neuroscience. 2008;151(4):1198-1207. [PMID:18207647] http://dx.doi.org/10.1016/j.neuroscience.2007.11.020

35. Dikmen SS, Corrigan JD, Levin HS, Machamer J, Stiers W, Weisskopf MG. Cognitive outcome following traumatic brain injury. J Head Trauma Rehabil. 2009;24(6):430-38. [PMID:19940676] http://dx.doi.org/10.1097/HTR.0b013e3181c133e9

36. McAllister TW. Neurobiological consequences of traumatic brain injury. Dialogues Clin Neurosci. 2011;13(3): 287-300. [PMID:22033563]

37. Hamm RJ, Dixon CE, Gbadebo DM, Singha AK, Jenkins LW, Lyeth BG, Hayes RL. Cognitive deficits following traumatic brain injury produced by controlled cortical impact. J Neurotrauma. 1992;9(1):11-20. [PMID:1619672] http://dx.doi.org/10.1089/neu.1992.9.11

38. Vanderploeg RD, Schwab K, Walker WC, Fraser JA, Sigford BJ, Date ES, Scott SG, Curtiss G, Salazar AM, Warden DL; Defense and Veterans Brain Injury Center Study Group. Rehabilitation of traumatic brain injury in active duty military personnel and veterans: Defense and Veterans Brain Injury Center randomized controlled trial of two rehabilitation approaches. Arch Phys Med Rehabil. 2008;89(12):2227-38. [PMID:19061734] http://dx.doi.org/10.1016/j.apmr.2008.06.015

Submitted for publication April 3, 2012. Accepted in revised form August 28, 2012.

This article and any supplementary material should be cited as follows:

Riechers RG, Shuster JL, Bryan KJ, Burant CJ, Ball SL. Prior housing conditions and sleep loss may affect recovery from brain injury in rats: A pilot study. J Rehabil Res Dev. 2013;50(4):455-62.

http://dx.doi.org/10.1682/JRRD.2012.04.0061

ResearcherID/ORCID: Sherry L. Ball, PhD: C-99252013; Jaime L. Shuster, MA: C-9620-2013

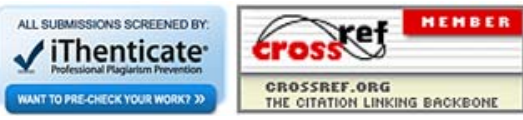

